\title{
LIGHT FOR BREVITY
}

- Anne L'Huillier - DOI: 10.1051/epn/2015503

n Department of Physics - Lund University

The shortest time interval controlled by a human being is the duration of a light pulse as short as only 100 attoseconds, i.e., $10^{-16} \mathrm{~s}$. This "attosecond" light pulse belongs to the extreme ultraviolet range (XUV) of the electromagnetic spectrum, with central photon energy typically between 20 and $200 \mathrm{eV}$. Related to its brevity, an attosecond pulse has a broad bandwidth covering tens of $\mathrm{eV}$. These are natural time and energy scales to study electron dynamics in atoms and molecules. 


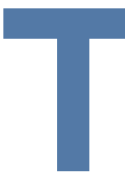

his feature article introduces the physics of attosecond pulses generated through high-order harmonic generation in gases to a general readership. We will also try to answer the question: what can we measure with such short pulses and what can we learn that we don't already know.

\section{Brief historical background}

In the late 80 s, scientists observed the emission of high-order harmonics of an intense laser focused into a gas cell [1,2]. Not only did the intensity of these harmonics not decrease with their order, forming a "plateau", but their number was high, reaching more than one hundred and thus covering a large bandwidth. It was suggested that if all these harmonics were emitted "in phase" their interference would lead to the formation of very short pulses. This suggestion was rapidly supported by a theoretical understanding of the single atom response to a strong laser field (Box 1) [3,4], as well as of propagation effects in the nonlinear medium (Box 2) [5].It took, however, almost fifteen years to demonstrate experimentally the existence of these ultrashort pulses. In 2001, the first train of attosecond pulses was observed at the Laboratoire d'Optique Appliquée in France [6], while the first isolated attosecond pulse was produced and measured at the Technical University of Vienna [7]. Since then, the number and diversity of attosecond sources, as well as their applications, is expanding worldwide [8].

\section{BOX1: SINGLE ATOM RESPONSE} -THE THREE STEP MODEL
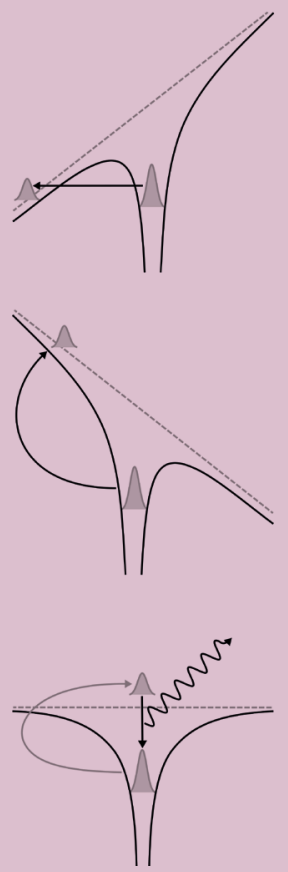

The laser field induces a distortion of the atomic potential so that an electron can be ionized by tunnel effect. When the laser field changes sign, the electron is attracted towards the ionic core. It may return with a high kinetic energy and be captured by its parent-ion. The electron recombines with the remaining ground state and an XUV photon is emitted. Everything happens within a laser optical cycle, $\sim$ one femtosecond $\left(10^{-15} \mathrm{~s}\right)$ and the emitted light pulse has a width of $\sim 200$ as. This coherent phenomenon follows the oscillations of the laser field and is repeated at each optical half cycle. Interference between consecutive attosecond pulses results in a frequency comb of high-order (odd) harmonics.

\section{Versatility of attosecond sources}

An interesting aspect of attosecond sources based upon high-order harmonic generation (HHG) in gases is their versatility. The radiation consists of a frequency comb of high-order harmonics, corresponding in the time domain to a train of attosecond pulses. It is possible to select a single harmonic, to generate a single attosecond pulse or to use the HHG frequency comb, thus keeping a high frequency resolution while benefiting from the underlying attosecond time structure. Single attosecond pulses can be generated using extremely short laser pulses (below $5 \mathrm{fs}$ ) in combination with spectral selection of the high-energy part of the spectrum [7]. It is also possible to manipulate the laser polarization (polarization gating) [9] and to spatially streak the attosecond pulse train emission (lighthouse [10] and noncollinear optical gating techniques [11]).

The performances of attosecond sources vary by orders of magnitude depending on the lasers used to drive them. Figure 1 (graph) shows the energy per harmonic pulse around $20 \mathrm{eV}$ as a function of the laser repetition rate. The diagonals indicate the average harmonic power. The take-home message of this picture is that harmonics can be generated with similar conversion efficiency (about $10^{-5}$ in this case) both using high-energy lasers at low repetition rate and high-average-power lasers, with a few $\mu \mathrm{J}$ laser energy at $\mathrm{MHz}$ repetition rate. $\mathrm{HHG}$ sources can be vastly different as shown in the photographs of Fig. 1 and should be designed for specific applications. There is also a lot of effort being devoted to the extension of HHG sources to higher photon energies, in the soft $\mathrm{X}$-ray range, in particular using mid-IR sources [12].

\section{Probing ultrafast dynamics}

The pulse duration and photon energy of attosecond pulses correspond to the characteristic time and energy of excitation processes in atoms and molecules. Heisenberg's principle gives for example a time $\tau=\hbar / \Delta \mathrm{E} \approx 60$ as for an electronic transition with energy $\Delta \mathrm{E}=10 \mathrm{eV}$. One could think of attosecond pulses as flashes of a camera filming the ultrafast motion of electrons in matter. The absorption of a pulse starts a process (for example, ionization or chemical reaction) and the absorption of a second pulse, delayed relative to the first, stops it. By varying the delay between the pump and probe, the movement can be reconstructed. However, this classical image of a pump/probe technique should be modified since the moving electrons are also waves or more precisely wave packets (wave-particle duality). The measurement of ultrashort electron dynamics which is made possible by attosecond light pulses in general implies the determination of the amplitude and phase of electronic wavepackets, which can be done either in the time domain, or equivalently, in the spectral domain. One of the techniques used for such phase and amplitude measurements, based upon interferometry, is illustrated in Box 3. It was first developed for the characterization of 
attosecond pulses and dubbed RABITT (Reconstruction of Attosecond Bursts by Interference of Two-photon Transitions) [6]. The technique often used for the measurement of single attosecond pulses, called streaking, is also based upon cross correlation between the XUV pulse and the fundamental field, however, at higher laser intensity [8]. In both cases, phase and amplitude measurements reflect the properties of the exciting field and of the ionization process used in the measurement. The characterization of attosecond pulses requires either to neglect the influence of ionization or to assume it known from other measurements or theoretical calculations. Conversely, the ionization dynamics can be determined if the attosecond pulses are well known or if their influence can be accounted for in the measurement. We illustrate below one fascinating application of attosecond pulses, the measurement of photoionization (or photoemission) time delays [13-15], opening a new area of research, which we called "ultrafast atomic and molecular physics", by analogy with ultrafast optics.

\section{Ultrafast atomic and molecular physics}

In ultrafast optics, an ultrashort pulse going through a dispersive medium will acquire a group delay due to the fact that the different frequency components of the pulse travel through the medium at different velocities. It can be calculated as the derivative of a spectral phase, dependent on the dispersion and length of the medium. Similarly, an electronic wavepacket created by absorbing an attosecond pulse will acquire a group delay when propagating through the atomic or molecular potential. This delay is the derivative with respect to energy of the phase of the photoionization probability amplitude.

The interferometric method presented in Box 3 allows us to determine the variation of this group delay across the spectrum of the attosecond pulses and/or to compare different ionization mechanisms if they are initiated by the same pulse. Figure 2 shows for example measurements of the difference of photoionization time delays in the $3 \mathrm{~s}$ and $3 p$ shell of argon. So far, we have "only" been able to answer simple questions such as: which of the 3 s or $3 p$ electron

\section{BOX2: MANY-ATOM RESPONSE - PROPAGATION IN THE NONLINEAR MEDIUM}

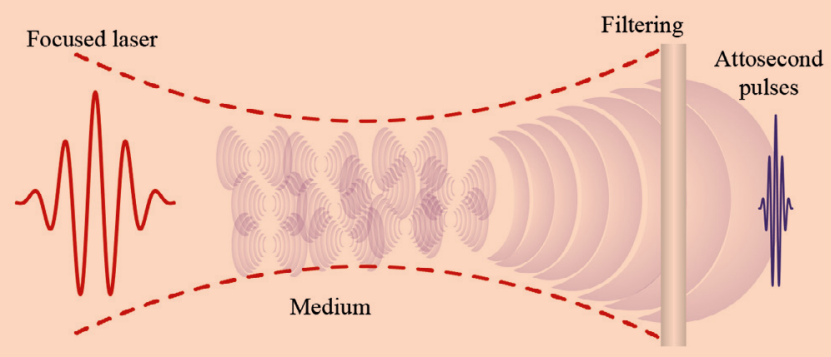

A laser is focused into a gas medium and induces a strong nonlinear atomic response. The emission from all the emitters adds coherently along the laser propagation direction. Phase matching depends on four contributions, the neutral gas dispersion, the dispersion induced by the presence of free electrons in the medium, the Gouy phase variation due to laser focusing, and the intrinsic dipole response. In general, the phase velocity at harmonic frequencies is superluminal since the refractive index above the ionization threshold is below one. A few percent ionization in the medium is necessary (and sufficient) to ensure that the phase velocity of the fundamental field matches that of the harmonic field. A filter is often placed in the beam to eliminate the fundamental field. Finally a detection gas is used for characterization as well as for applications (Courtesy of Marcus Dahlström).

wavepacket is coming first out of the potential [14] or how long does it take for two electrons to be ejected [16]. With improved spectral range and temporal precision, we will get much more insight into the dynamics of correlated electrons in matter, e.g. close to resonances $[17,18,19]$

In conclusion, one of the most interesting applications of attosecond pulse technology might be the possibility to measure phases (or phase derivatives) of quantum mechanical probability amplitudes. Such measurements, combined with intensity (or cross section) determinations allow us to

V FIG. 1: Left: Long (15 m) attosecond beamline driven by a $200 \mathrm{~mJ}$ 40-fs 10-Hz Ti-Sapphire laser system. Middle: Compact, table-top, $200 \mathrm{kHz}-$ repetition rate attosecond source driven by an OPCPA laser system. Right: Graph showing the average power reached in one harmonic at 20-30 eV photon energy (grey). The experimental measurements (blue dots) are from (left to right) E. Takahashi et al., Optics Lett. 27, 1920 (2002); E. Constant et al., Phys. Rev. Lett. 82, 1668 (1999); S. Hädrich et al., Nat. Phot. 8, 779 (2014); S. Hädrich et al., Light Science and applications 4, e320 (2015) (Courtesy of Christoph Heyl).
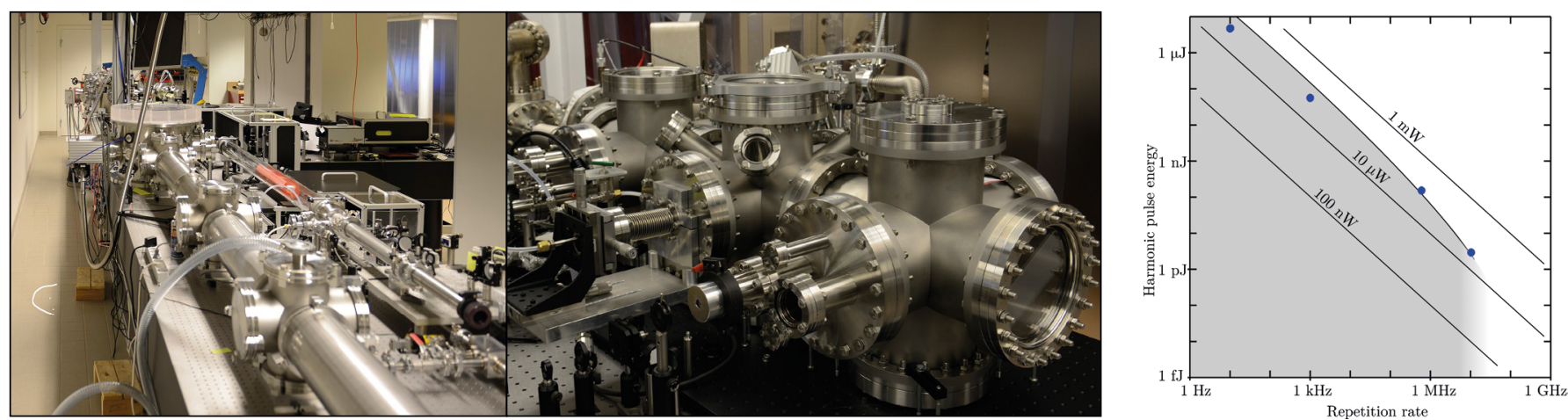


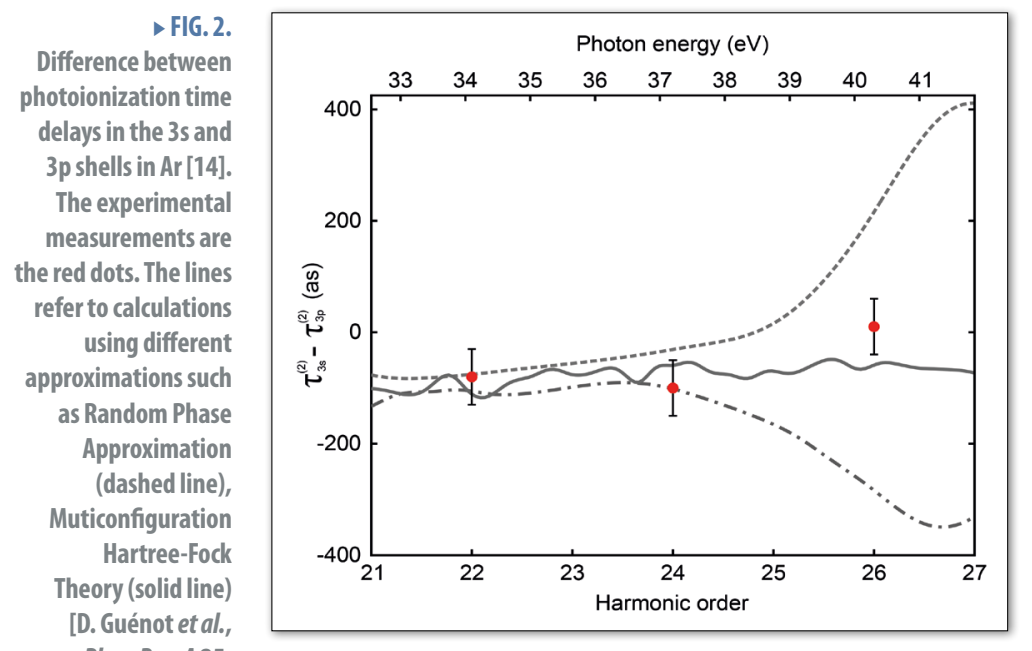

Phys. Rev. A 85 , 053424(2012)],

Time Dependent

Density Functiona

Theory (dot-dashed

line) [G. Dixit et al.

Phys. Rev. Lett. 111,

203003 (2013)].

These calculations

show quite different

results at high energy

$(40 \mathrm{eV})$ close to the

Cooper minimum of

the 3 s shell where

correlation effects

between the $3 s$ and

$3 p$ subshells are

important. Note that

the indicated values

need to be corrected

for the influence of

the IR field to deduce

the photoionization

time delays. (Courtesy

of Diego Guénot

and David Kroon)

completely characterize electron wavepackets in the spectral or temporal domain, giving thus access to the temporal dynamics of the electron wavepacket. The door is now open to the investigation of more complex problems such as the dynamics of electrons in inner shells or the transfer of charge in molecules [20].

\section{Aknowledgment}

The author acknowledges support from the European Research Council, the Swedish Research Council and the Knut and Alice Wallenberg Foundation.

\section{About the author}

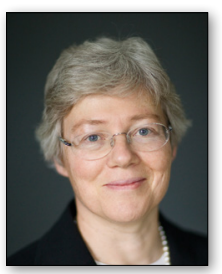

Anne L'Huillier is professor in Atomic Physics at Lund University since 1997, and leader of a research group in attosecond science. After a $\mathrm{PhD}$ at the University Pierre et Marie Curie in 1986, she was researcher at the Commissariat à l'Energie Atomique, Saclay, France until 1995 when she moved to Sweden. She has made numerous contributions to the field of high-order harmonic generation in gases and its application to attosecond pulse generation. She is member of the Royal Swedish Academy of Sciences since 2004

\section{References}

[1] A. McPherson et al., J. Opt. Soc. Am. B 4, 595 (1987)

[2] M. Ferray et al., J. Phys. B 21, 31 (1988)

[3] K. J. Schafer et al., Phys. Rev. Lett. 70, 1599 (1993)

[4] P. B. Corkum, Phys. Rev. Lett. 71, 1994 (1993)

[5] A. L'Huillier, K. J. Schafer and K. C. Kulander, Phys. Rev. Lett. 66 , 2200 (1991)
[6] P. M. Paul et al., Science 292, 1689 (2001)

[7] M. Hentschel et al., Nature 414, 509 (2001)

[8] F. Krausz and M. Ivanov., Rev. Mod. Phys. 81, 163 (2009)

[9] I. J. Sola et al., Nature Physics 2, 319 (2006)

[10] H. Vincenti and F. Quéré, Phys. Rev. Lett. 108, 113904 (2012)

[11] M. Louisy et al., Optica 2, 563 (2015)

[12] T. Popmintchev et al. Science 336, 1287 (2012)

[13] M. Schultze et al., Science 328, 1658 (2010)

[14] K. Klünder et al., Phys. Rev. Lett. 106, 143002 (2011)

[15] R. Pazourek, S. Nagele and J. Burgdörfer, Rev. Mod. Phys. 87, 765 (2015)

[16] E. Månsson et al., Nature Physics 10, 207211 (2014)

[17] M. Drescher et al., Nature 419, 803 (2002)

[18] C. Ott et al., Science 340, 716 (2013)

[19] P. Tzallas et al., Nature Physics 7, 781 (2011)

[20] F. Calegari et al., Science 346, 336 (2014)

\section{BOX3: ATTOSECOND INTERFEROMETRY}

An optical Mach Zendher interferometer is used to generate attosecond pulse trains together with a small fraction of the infrared (IR) laser field used for the generation, with a variable time delay. Both XUV and IR fields are focused into a detection gas including an electron spectrometer. Ionization of the gas by the HHG frequency comb leads to electron peaks with energy equal to $n \hbar \omega-I_{p}$, where $n$ is an odd integer and $l_{p}$ the ionization energy. The absorption or emission of an IR photon leads to sideband peaks at energies $n \hbar \omega-I_{p}$, with $n$ even. Because two quantum paths lead to the same final state, the probability amplitudes interfere and the electron signal oscillates as a function of delay between the XUV and IR pulses. Our measurement consists in measuring the phase of these oscillations,
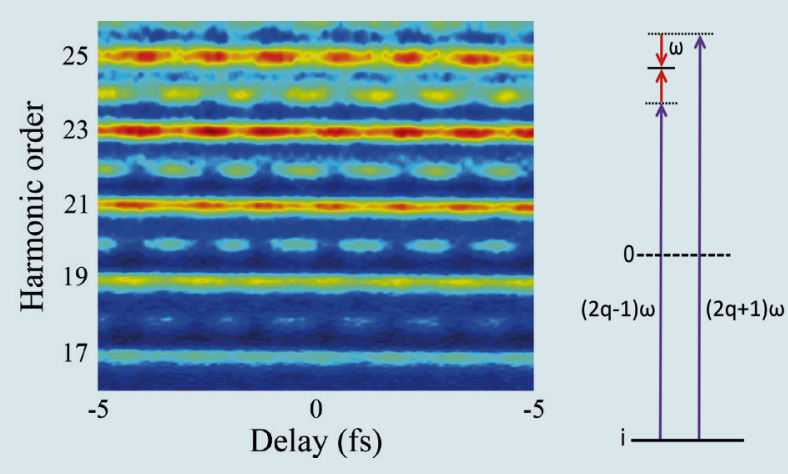

as a function of energy. The measured phase offset is the phase difference of the complex amplitudes of the two quantum paths leading to the same final state. This includes the phase difference between consecutive harmonics as well as a phase difference induced by the two-photon ionization process. 